\title{
Exponential stability of implicit numerical solution for nonlinear neutral stochastic differential equations with time-varying delay and Poisson jumps
}

\author{
Haoyi $\mathrm{Mo}^{1}$, Linna $\mathrm{Liu}^{2}$, Mali Xing ${ }^{1}$, and feiqi Deng ${ }^{3}$ \\ ${ }^{1}$ Guangdong University of Technology \\ ${ }^{2}$ Zhongyuan University of Technology \\ ${ }^{3}$ South China University of Technology
}

May 23, 2020

\begin{abstract}
The aim of this work is to investigate the exponential mean-square stability for neutral stochastic differential equations with time-varying delay and Poisson jumps. We give some conditions that all the drift, diffusion and jumps coefficients can be nonlinear, to obtain the stability of the analytic solution. It is revealed that the implicit backward Euler-Maruyama numerical solution can reproduce the corresponding stability of the analytic solution under these nonlinear conditions. This is different from the explicit Euler-Maruyama numerical solution whose stability depends on the linear growth condition. With some requirements related to the delay function and the property of compensated Poisson process, we deal with time-varying delay and Poisson jumps. One highly nonlinear example is provided to confirm the effectiveness of our theory.
\end{abstract}

\section{Hosted file}

Main pdf-Mathematical Methods.pdf available at https://authorea.com/users/325517/articles/ 453513-exponential-stability-of-implicit-numerical-solution-for-nonlinear-neutralstochastic-differential-equations-with-time-varying-delay-and-poisson-jumps 
figures/vary1/vary1-eps-converted-to.pdf 
figures/vary2/vary2-eps-converted-to.pdf 
figures/vary3/vary3-eps-converted-to.pdf 
figures/vary4/vary4-eps-converted-to.pdf 